\title{
Influence of the particle injection rate, droplet size distribution and volume flux angular distribution on the results and computational time of water spray CFD simulations
}

\author{
TAREK BEJI ${ }^{1}$, SETAREH EBRAHIM ZADEH ${ }^{1}$, GEORGIOS MARAGKOS ${ }^{1}$ and BART MERCI ${ }^{1}$ \\ ${ }^{1}$ Department of Flow, Heat and Combustion Mechanics \\ Ghent University - UGent \\ B-9000, Ghent, Belgium
}

\begin{abstract}
The paper presents a detailed sensitivity analysis on the volume flux probability density function (PDF) to represent water spray patterns with computational fluid dynamics (CFD). The effects of the turbulent viscosity model and the cell size are also investigated. The test case considered herein is a $30^{\circ}$ full cone water mist spray emerging from a nozzle that operates at a pressure of $750 \mathrm{kPa}$ and delivers a water flow rate of $0.084 \mathrm{lpm}$. The errors solely induced by the limited number of computational droplets per second, $N_{p}$, are proportional to $1 / \sqrt{N_{p}}$ and could reach up to $35 \%$. The computational time generally increases linearly with $N_{p}$. The paper illustrates also the better numerical performance of the lognormal-Rosin-Rammler droplet size distribution over the Rosin-Rammler distribution, especially in terms of reaching a converged volume-median diameter with increased $N_{p}$. Furthermore, a uniform angular distribution is shown to provide results in better agreement with experimental data than a Gaussian-type distribution for the case at hand. For a sufficiently fine grid, the dynamic Smagorinsky and the modified Deardorff models converge to similar radial profiles of the water volume flux at $300 \mathrm{~mm}$ from the nozzle, with a deviation of less than $6 \%$ from the experiments. The deviations for the volume-median diameter are about $50 \%$ in the core region of the spray.
\end{abstract}

KEYWORDS: CFD; modeling; suppression.

\section{NOMENCLATURE LISTING}

$\begin{array}{ll}A & \text { surface area }\left(\mathrm{m}^{2}\right) \\ C & \text { constant } \\ C_{d} & \text { drag coefficient } \\ c & \text { specific heat }(\mathrm{kJ} / \mathrm{kg} . \mathrm{K}) \\ D & \text { diameter }(\mathrm{m}) \\ D & \text { mass diffusion coefficient }\left(\mathrm{m}^{2} / \mathrm{s}\right) \\ F & \text { cumulative fraction } \\ f & \text { probability density function } \\ g & \text { gravitational acceleration }\left(\mathrm{m} / \mathrm{s}^{2}\right) \\ h & \text { heat transfer coefficient }\left(\mathrm{kW} / \mathrm{m}^{2} / \mathrm{K}\right) \\ h_{m} & \text { mass transfer coefficient }(\mathrm{m} / \mathrm{s}) \\ h_{v} & \text { heat of vaporization }(\mathrm{kJ} / \mathrm{kg}) \\ m & \text { mass }(\mathrm{kg}) \\ N_{p} & \text { particle injection rate }(1 / \mathrm{s}) \\ n & \text { number of real droplets } \\ P & \text { pressure }(\mathrm{Pa}) \\ R e & \text { Reynolds number } \\ r_{0} & \text { radius of the spherical boundary }(\mathrm{m}) \\ S c & \text { Schmidt number } \\ T & \text { temperature }(\mathrm{K}) \\ t & \text { time }(\mathrm{s}) \\ u & \text { velocity }(\mathrm{m} / \mathrm{s}) \\ V & \text { volume }\left(\mathrm{m}^{3}\right) \\ \dot{V} & \text { volume flux }(\mathrm{m} / \mathrm{s}) \\ & \end{array}$

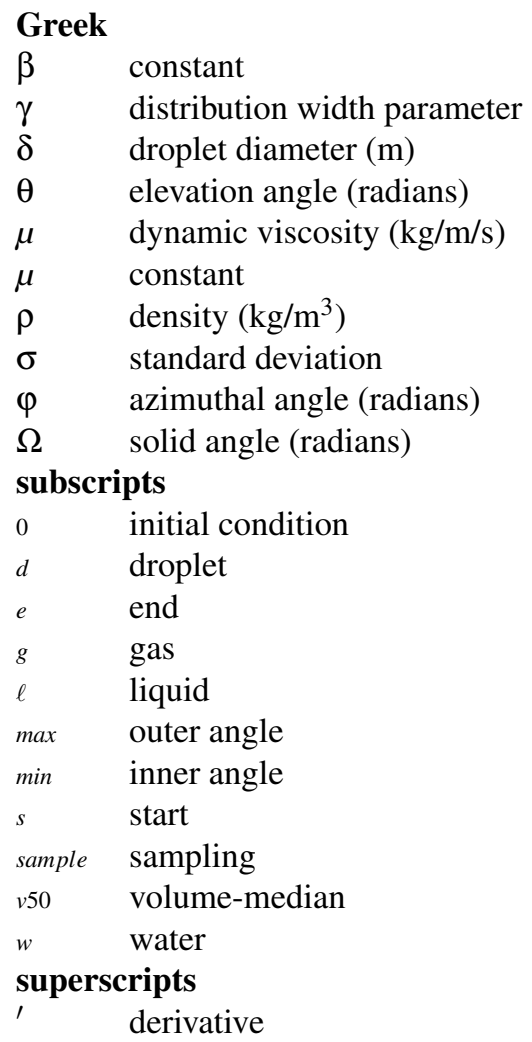




\section{INTRODUCTION}

Water sprays are widely used for fire control and suppression. Several experimental programs have been developed in order to examine their dynamics and effectiveness for a variety of fire scenarios (e.g., penetration through a fire plume [1] or interaction with a ceiling vent [2]). The continuing advances in numerical modelling offer a valuable support tool to experimental testing for design purposes by exploring a wider range of fire scenarios at reduced cost. However, insights provided by numerical modelling (e.g., Computational Fluid Dynamics, CFD) can only be valuable if modelling is proven (through validation studies) to be sufficiently accurate. The capabilities of a CFD code to predict a water spray pattern are generally assessed first in the absence of any fire-induced flow (e.g., fire plume or a ceiling jet) in order to avoid the complexities that arise from the strong coupling between the gas phase and the liquid phase. The assessment (validation) exercise is performed against nozzle characterization experiments that provide local measurements of water volume flux, droplet velocities and droplet diameters within the spray (e.g., [1,3]).

The first complexity in water spray simulations stems from the limited ability to predict sprinkler atomization, i.e., the process of breaking up a volume of liquid into small drops [4]. In order to by-pass this complexity, water droplets are introduced into the computational domain at a prescribed distance, $r_{0}$, from the nozzle using a Eulerian-Lagrangian approach. As a result, the spray boundary consists of a spherical surface defined by $r_{0}$ and two angles, namely the elevation angle $\theta$ and the azimuthal angle $\varphi$. The associated spray boundary conditions are essentially related to (1) the water volume flux distribution across the spray solid angle, and (2) the droplet size distribution. Furthermore, since it is impractical to simulate the motion of every droplet in the sprinkler spray, a particle injection rate, $N_{p}$ must be prescribed. Each computational Lagrangian particle represents a large group of real droplets. In [3,5], the concentration of particles is assumed to be higher in the core region of the spray. A Gaussian profile is used in [3], with a spread factor $\beta=5$ calibrated against water mist spray measurements. In this paper, we intend to study the influence of this parameter for the water mist spray characterized in [1]. Regarding the droplet size distribution, it is generally represented by either a Rosin-Rammler function or a combination of a lognormal and Rosin-Rammler functions [6,7]. The differences between the two options are examined in this paper. Finally, the effect of $N_{p}$ on the accuracy of the simulations will be examined. In [3] a value of $N_{p}=2 \times 10^{5} s^{-1}$ has been used. The second value that was tested, $N_{p}=4 \times 10^{5} s^{-1}$, did not yield substantially different results. We provide in this paper a more complete sensitivity study on this parameter for the water spray characterized in [1] and verify that the error induced by the limited number of Lagrangian particles is proportional to $1 / \sqrt{N_{p}}$ as suggested in [8]. The evolution of the CPU time as a function of $N_{p}$ is also examined in order to find a good compromise between computational cost and accuracy.

The work described in this paper lays the groundwork for the numerical simulations of the interaction between the studied water mist spray and hot air jets. This configuration has been examined experimentally in [1] where three regimes have been identified: a first regime where the spray momentum is dominant, a second regime where the hot air jet momentum is dominant, and a third intermediate regime where both momenta are comparable, leading to a stagnation plane (i.e., interaction boundary) located at around mid-way between the nozzle and the hot air inlet. The comprehensive data described in [1] constitutes an excellent database for CFD code validation.

\section{MATHEMATICAL MODELS}

The computational fluid dynamics (CFD) package used in this study is the Fire Dynamics Simulator, FDS 6.1.2 (SVN Revision No. 20564) [6,7]. Turbulence is described in FDS using Large Eddy Simulation (LES). Unless specified otherwise, turbulent viscosity is modeled here using the Dynamic Smagorinsky approach. However, other approaches are available, such as the modified Deardorff model (default model in FDS). The liquid phase is modelled using a Lagrangian approach. The governing equations and the solution methods for both the gas and liquid phase are described in [7]. Only the most essential aspects in spray modelling are repeated here for the sake of clarity. 


\section{Spray injection model}

Due to the limited ability to predict sprinkler atomization in CFD simulations, water droplets are introduced into the computational domain at a prescribed distance, $r_{0}$, from the nozzle, using a Eulerian-Lagrangian approach. As a result, the spray boundary consists of a spherical surface defined by $r_{0}$ and two angles, namely the elevation angle $\theta(\theta=0$ for the vertical direction) and the azimuthal angle $\varphi$. Droplets are assumed to emerge from the surface $r_{0}(\theta, \varphi)$ with an initial velocity calculated as [3]:

$u_{d, 0}=C \sqrt{\frac{\Delta P_{w}}{\rho_{d}}}$

where $\rho_{d}$ is the water density and $\Delta P_{w}$ the pressure at which the nozzle is operating. The constant $C$ is a factor that accounts for friction losses in the nozzle, estimated in [3] as $C=0.95$.

Furthermore, the droplets emerge according to a probability density function, $f_{v}$, based on the volume flux through a spherical surface at $r_{0}$ and defined as [8]:

$\int_{\left[\theta, \varphi, D_{d}\right]} f_{v}\left(\theta, \varphi, D_{d}\right) r_{0}^{2} d \Omega d D_{d}=1$

where $d \Omega$ is the differential solid angle defined as [8]:

$d \Omega=\sin (\theta) d \theta d \varphi$

The probability density function $f_{v}$ may be decomposed into conditional probabilities as [8]:

$f_{v}\left(\theta, \varphi, D_{d}\right)=f_{v, \Omega}(\theta, \varphi) f_{v, D_{d}}\left(D_{d} \mid \theta, \varphi\right)$

where $f_{v, \Omega}(\theta, \varphi) d \theta d \varphi$ is the probability of finding volume flux from any drops emerging in between the angular coordinates $(\theta, \varphi)$ and $(\theta+d \theta, \varphi+d \varphi)$, and $f_{v, D_{d}}\left(D_{d} \mid \theta, \varphi\right) d D_{d}$ is the probability of finding volume flux from drops of size $D_{d}$ to $D_{d}+d D_{d}$ given an angular location $(\theta, \varphi)$.

The volume flux angular probability density distribution, $f_{v, \Omega}(\theta, \varphi)$, is decomposed into the marginal PDFs, assuming statistical independence of $\theta$ and $\varphi$ :

$f_{v, \Omega}(\theta, \varphi)=f_{v, \Omega}(\theta) f_{v, \Omega}(\varphi)=\frac{1}{2 \pi} f_{v, \Omega}(\theta)$

where the volume flux is uniformly distributed over $\varphi$ and varies with $\theta$ as [3,6]:

$f_{v, \Omega}(\theta)=\exp \left[-\beta\left(\mu-\frac{\theta-\theta_{\min }}{\theta_{\max }-\theta_{\min }}\right)^{2}\right]$

where $\beta$ is a spread parameter (the default value in [6] is $\beta=5$ ) and $\mu$ is a parameter that gives the location in the spray at which most of the water is released. By default $\mu=0$, indicating that most of the water is released in the core region (i.e., axis) of the spray. If $\beta=0$ the distribution is said to be uniform. The angles $\theta_{\min }$ and $\theta_{\text {max }}$ delimit the inner and outer boundary of the spray. 
The initial droplet size distribution of the water spray is expressed in terms of its Cumulative Volume Fraction (CVF), which is often assumed to fit the Rosin-Rammler distribution [9]:

$1-F_{v, D_{d}}\left(D_{d}\right)=\exp \left[-\left(\frac{D_{d}}{\bar{D}_{d}}\right)^{\gamma}\right]$

where $1-F_{v, D_{d}}\left(D_{d}\right)$ is the volume fraction of water in droplets of diameter greater than $D_{d}, \bar{D}_{d}$ is a size parameter and $\gamma$ is a distribution width parameter [9].

Equation (5) is often rewritten as:

$F_{v, D_{d}}\left(D_{d}\right)=1-\exp \left[-\ln (2)\left(\frac{D_{d}}{D_{v 50}}\right)^{\gamma}\right]$

where $D_{v 50}$ is the volume-median diameter $(\mathrm{VMD})$ calculated as $D_{v 50}=\bar{D}_{d}(\ln (2))^{1 / \gamma}$ [9]. The VDM denotes that half of the cumulated water volume is represented by droplets having a diameter smaller than $D_{v 50}$.

The PDF of the volume fraction (i.e., the volume fraction distribution (VFD)) is calculated as the first derivative of the function $F_{v, D_{d}}\left(D_{d}\right)$.

$f_{v, D_{d}}\left(D_{d}\right)=\frac{d F\left(D_{d}\right)}{d D_{d}}=\frac{\ln (2) \gamma D_{d}^{\gamma-1}}{D_{v 50}^{\gamma}} \exp \left[-\ln (2)\left(\frac{D_{d}}{D_{v 50}}\right)^{\gamma}\right]$

The corresponding PDF for the droplet diameter (i.e., the number fraction distribution (NFD)) is defined as:

$f_{N}\left(D_{d}\right)=\frac{f_{v, D_{d}}\left(D_{d}\right)}{D_{d}^{3}} / \int_{0}^{\infty} \frac{f_{v, D_{d}}(\delta)}{\delta^{3}} d \delta$

Droplets are randomly selected by equating the Cumulative Number Fraction (CNF) with a uniformly distributed random variable $F_{N, D_{d}}$ :

$F_{N, D_{d}}\left(D_{d}\right)=\int_{0}^{D_{d}} f_{N, D_{d}}(\delta) d \delta$

The sequence of Eqs. (8) to (11) show how the droplet diameter distribution is connected to the CVF. Figure 1 shows an example of the outcome of Eqs.(8) to (11) for $D_{v 50}=60 \mu \mathrm{m}$ and two values for the distribution width parameter, $\gamma=2$ and $\gamma=4$. More particularly, Fig. 1d shows that for $\gamma=2$ the curve approaches the ordinate axis asymptotically as the droplet size decreases. This is explained mathematically by combining Eqs. (9) and (10), which yields $f_{N}\left(D_{d}\right) \sim D_{d}^{\gamma-4}$ and means that unimodal number density curves only exist when $\gamma>4$ [9]. Therefore, when a Rosin-Rammler distribution is prescribed with a width parameter $\gamma<4$, upper and lower limits for the droplet size are prescribed $\left(D_{d, \max }\right.$ and $\left.D_{d, \min }\right)$, the $D_{d \text {,min }}$ being more critical

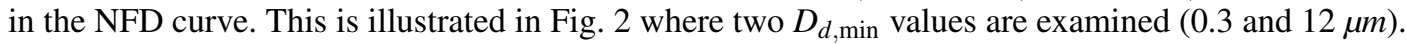

As pointed out in [9], if the lower limit of integration (in Eq. (10)) is decreased, the value of the VMD will be substantially reduced (as the limit of integration is reduced to zero the area under the NFD curve 

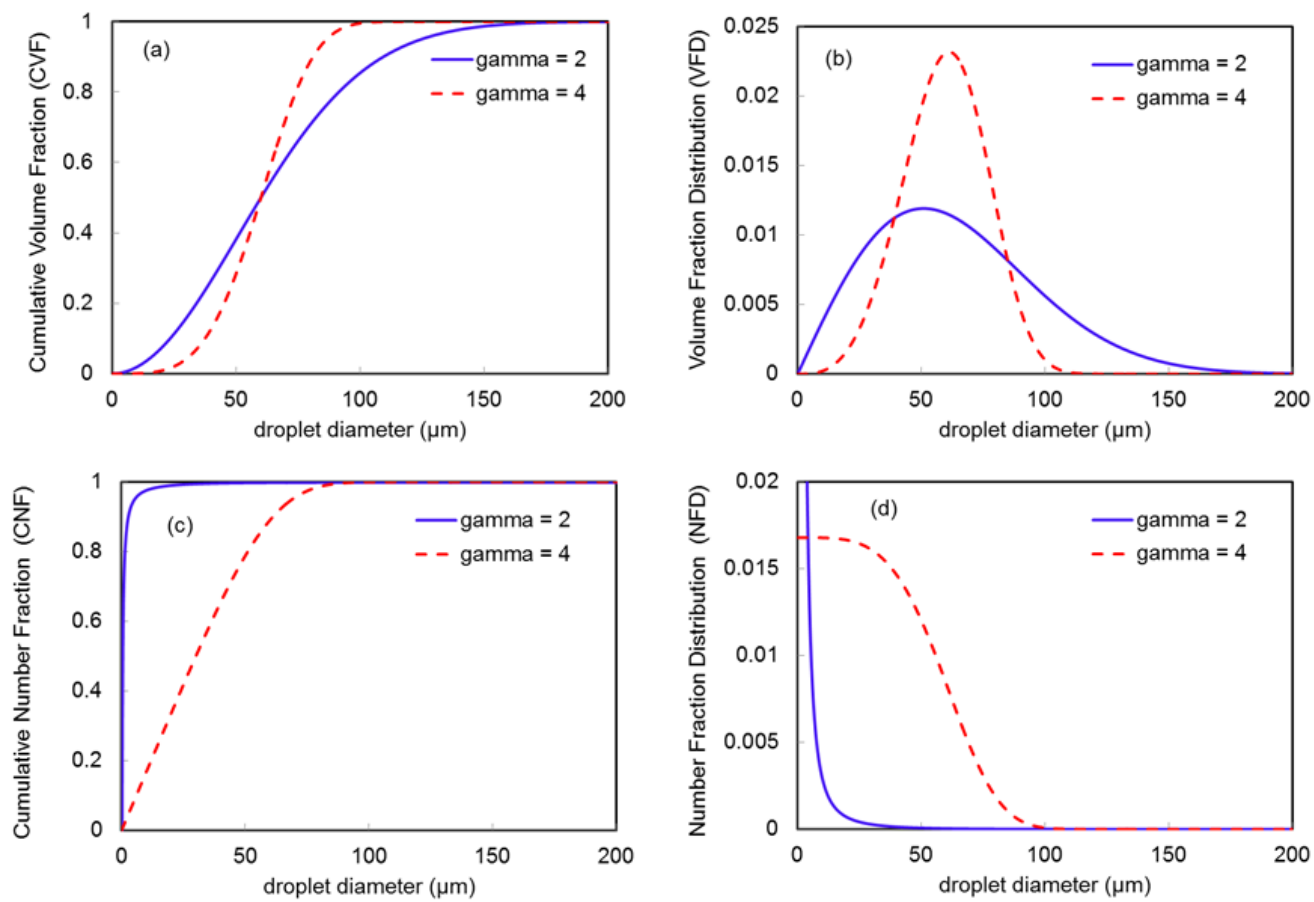

Fig. 1. Rosin-Rammler droplet size distribution for $D_{v 50}=60 \mu \mathrm{m}$ and two different values for the distribution with parameter, i.e., $\gamma=2$ and $\gamma=4$. (a) Cumulative Volume Fraction. (b) Volume Fraction Distribution. (c) Cumulative Number Fraction. (d) Number Fraction Distribution (with $D_{d \text {,min }}=0.3 \mu m$ ).
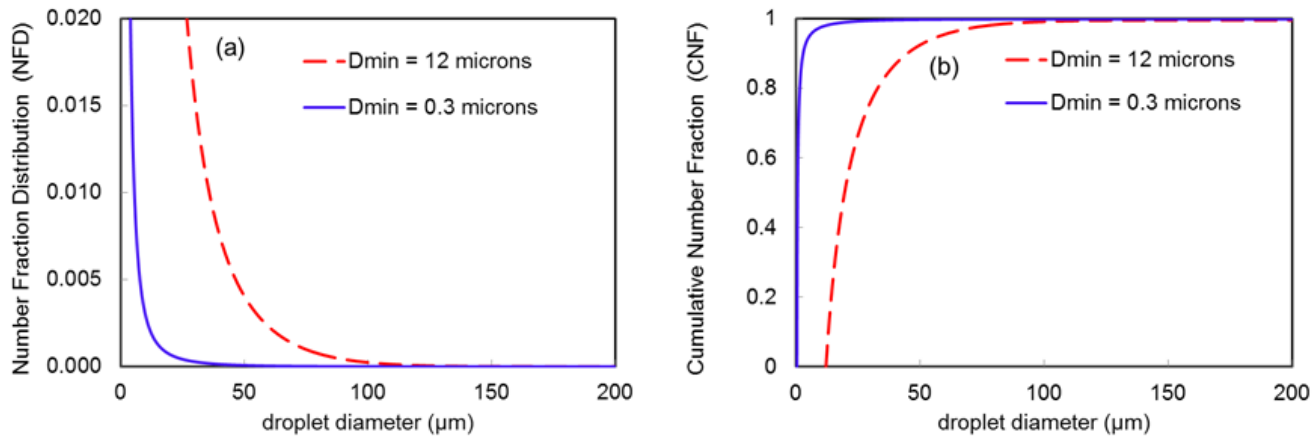

Fig. 2. Influence of the minimum droplet size in the Rosin-Rammler distribution (with $D_{v 50}=60 \mu m$ and $\gamma=2$ ) on the (a) Number Fraction Distribution and (b) Cumulative Number Fraction.

becomes infinite). The divergence of the integrals at $D_{d} \rightarrow 0$ limit is a known drawback of the Rosin-Rammler distribution.

A way to by-pass the problem induced by the lower limit of integration in Eq. (10) is to prescibe a skew distibution such as the log-normal function for the small diameters [9]. Hence, the default approach in FDS is to use a combination of the Rosin-Rammler and lognormal distributions as follows:

$F_{v, D_{d}}\left(D_{d}\right)=\left\{\begin{array}{lr}\frac{1}{\sigma \sqrt{2 \pi}} \int_{0}^{D_{d}} \frac{1}{\delta} \exp \left(-\frac{\left[\ln \left(D_{d}\right)-\ln \left(D_{v 50}\right)\right]^{2}}{2 \sigma^{2}}\right) d \delta ; \quad D_{d} \leq D_{v 50} \\ 1-\exp \left[-\ln (2)\left(\frac{D_{d}}{D_{v 50}}\right)^{\gamma}\right] \quad ; \quad D_{d}>D_{v 50}\end{array}\right.$ 
where the width parameters are related by:

$\sigma=\frac{2}{\sqrt{2 \pi}(\ln (2)) \gamma}$

to ensure continuity at $D_{v 50}$.

Figure 3 illustrates the differences between a Rosin-Rammler distribution and a mixed distribution for $D_{v 50}=$ $60 \mu m$ and $\gamma=2$. A slight difference in the CVF profile might result in significant differences in CNF and NFD curves.
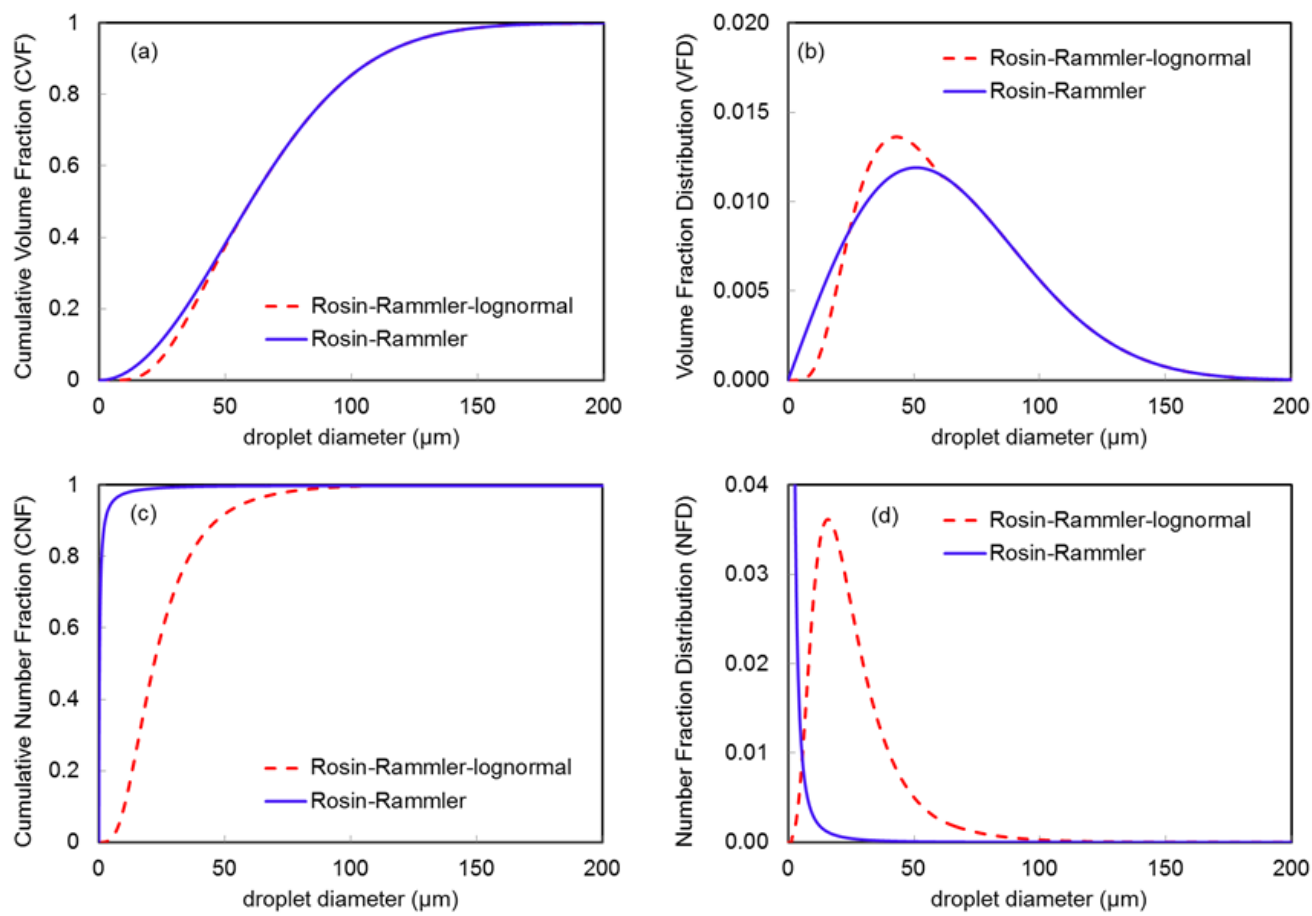

Fig. 3. Comparison between the Rosin-Rammler and Rosin-Rammler-lognormal droplet size distribution for $D_{v 50}=60 \mu m$. (a) Cumulative Volume Fraction. (b) Volume Fraction Distribution. (c) Cumulative Number Fraction. (d) Number Fraction Distribution (with $D_{d, \min }=0.3 \mu \mathrm{m}$ ).

\section{Lagrangian particle model}

The conservation equations of mass, momentum and energy are [6,7]:

$$
\frac{d m_{d}}{d t}=-A_{d} h_{m} \bar{\rho}\left(Y_{\ell}-\tilde{Y}_{g}\right)
$$

$\frac{d}{d t}\left(m_{d} \vec{u}_{d}\right)=-\pi \frac{D_{d}^{2}}{8} \bar{\rho} C_{d}\left(\vec{u}_{d}-\vec{u}\right)\left|\vec{u}_{d}-\vec{u}\right|-m_{d} \vec{g}$

$$
m_{d} c_{d} \frac{d T_{d}}{d t}=A_{d} h\left(\tilde{T}_{g}-T_{d}\right)+\frac{d m_{d}}{d t} h_{v}
$$


where $m_{d}, A_{d}, u_{d}, D_{d}, c_{d}$ and $T_{d}$ are respectively the droplet mass, surface area, velocity, diameter, specific heat and temperature. The droplets are assumed to be spherical. Thus, $A_{d}=\pi D_{d}^{2}$ and $m_{d}=\rho_{d}\left(\pi D_{d}^{3} / 6\right)$. The variables $\bar{\rho}, \tilde{T}_{g}, u$ and $\tilde{Y}_{g}$ denote respectively the resolved gas phase density, temperature, velocity and mass fraction of water in a cell occupied by the droplet. The variables $t, C_{d}, h, h_{m}, h_{v}, g$ and $Y_{\ell}$ denote respectively the time, the drag coefficient, the heat transfer coefficient between the liquid droplet and the gas, the mass transfer coefficient, the liquid heat of vaporization, gravitational acceleration and the liquid equilibrium vapor mass fraction. The latter (i.e., $Y_{\ell}$ ) is calculated using the Clausius-Clapeyron equation.

The mass transfer coefficient is calculated as:

$h_{m}=\frac{\left(2+0.6 \operatorname{Re}_{d}^{1 / 2} S c^{1 / 3}\right) D_{\ell g}}{D_{d}}$

where $S c$ is the Schmidt number (taken as $S c=0.6$ ) and $D_{\ell g}$ is the binary diffusion coefficient between the liquid vapor and the surrounding gas (see Eq.(4.31) in [7]). The droplet Reynolds number, $R e_{d}$, is calculated as:

$\operatorname{Re}_{d}=\frac{\bar{\rho}\left|\vec{u}_{d}-\vec{u}\right| D_{d}}{\mu}$

where $\mu$ is the dynamic gas phase viscosity.

The drag coefficient is calculated as:

$C_{d}=\left\{\begin{array}{lc}24 / \operatorname{Re}_{d} & \operatorname{Re}_{d}<1 \\ 24\left(0.85+0.15 \operatorname{Re}_{d}^{0.687}\right) / \operatorname{Re}_{d} & 1<\operatorname{Re}_{d}<1000 \\ 0.44 & 1000<\operatorname{Re}_{d}\end{array}\right.$

Furthermore, when the local droplet volume fraction exceeds $10^{-2}$ (i.e., dense spray) a reduction in drag is accounted for in FDS. This reduction is equal to the hydrodynamic force ratio of the trailing particle to an isolated particle. For the case that will be examined hereafter, the local droplet volume fraction did not exceed $10^{-3}$. Thus, the spray may be regarded as dilute and the drag reduction effect is negligible.

\section{CASE DESCRIPTION AND COMPUTATIONAL SETUP}

The test case considered herein is a $30^{\circ}$ full cone water mist spray emerging from a nozzle that operates at a pressure of $750 \mathrm{kPa}$ and delivers a water flow rate of $0.084 \mathrm{lpm}$ [1]. The experimental campaign carried out in [1] aims at characterizing the interactions between hot air plumes and water sprays and providing a comprehensive and well-documented database for CFD validation. The campaign comprises three test series: (1) a test characterizing the water spray, (2) three tests characterizing hot air flows impinging on a horizontal ceiling and (3) tests where the water spray and the hot air-driven flow are interacting. In this paper, we focus on the simulation of the water spray (i.e., first test) before addressing the simulations of the remainder of the experimental configurations in forthcoming publications. The measured volume-median diameter and the distribution width at $30 \mathrm{~mm}$ below the nozzle are respectively $D_{v, 50}=60 \mu \mathrm{m}$ and $\gamma=2$ [10]. The experiments were modeled using a rectangular computational area $0.4 \mathrm{~m}$ high, $0.2 \mathrm{~m}$ wide and $0.2 \mathrm{~m}$ deep. The computational area was open to flow on the sides and the bottom of the domain. The upper part of the domain has been modeled as a wall in order to represent the ceiling plate used in the experiments. The nozzle was placed at $30 \mathrm{~mm}$ from the ceiling. Figure 4 shows the computational set-up and the location of the measurement points. Unless specified otherwise, a cell size of $4 \mathrm{~mm}$ has been used. 

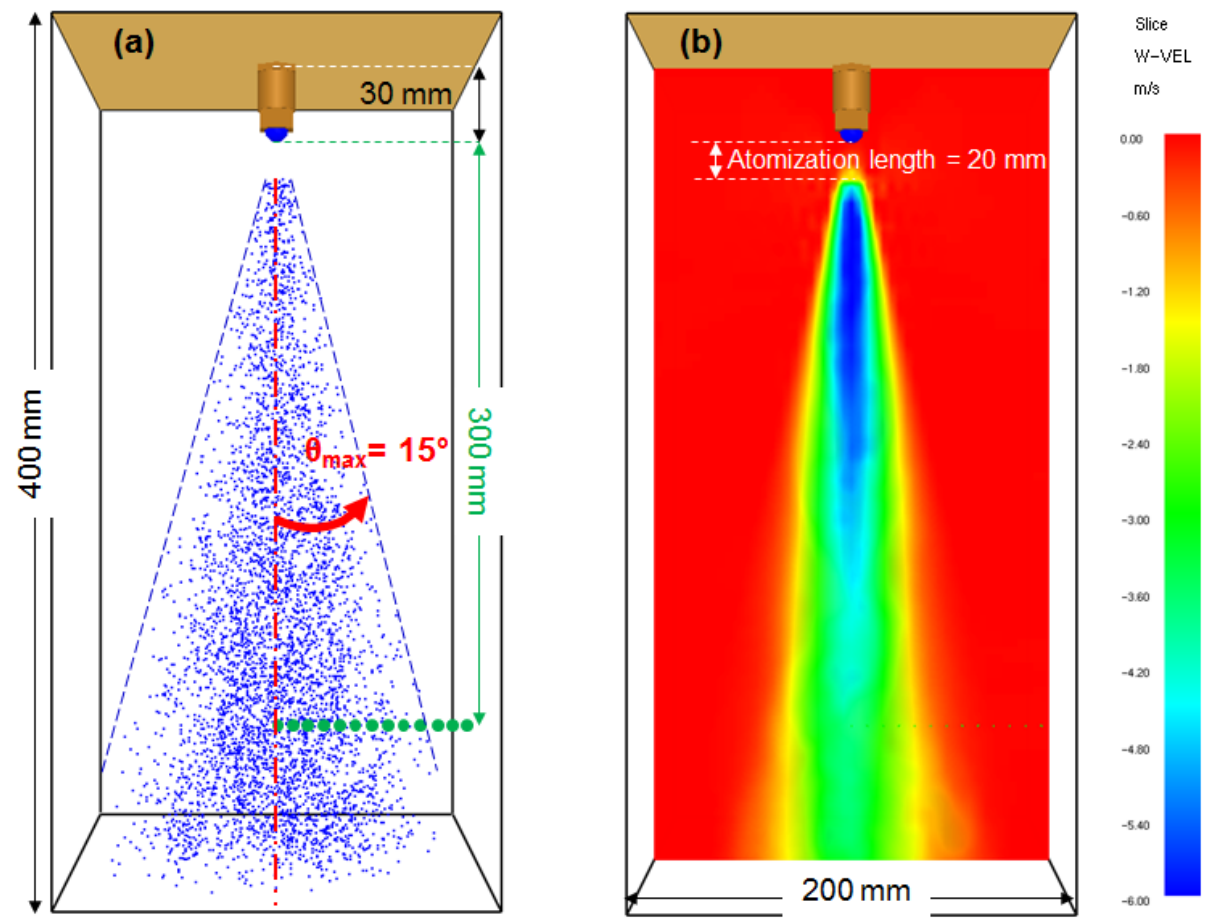

Fig. 4. Smokeview visualization of the computational set-up (dimensions, position of the nozzle, location of the measurements (green dots), water spray elevation angle, and atomization length). (a) Spray pattern. (b) Time-averaged velocity slice.

The time-integrated droplet volume flux in the vertical direction is calculated in FDS as:

$\dot{V}_{d}^{\prime \prime}=\frac{1}{t_{e}-t_{s}} \int_{t_{s}}^{t_{e}} \frac{\sum_{i=1}^{N_{p}} n_{i} \pi\left(D_{d, i}^{3} / 6\right) w_{d, i}}{V_{\text {sample }}} d t$

where $t_{s}$ and $t_{e}$ are respectively the start time and end time for the integration (taken here as $t_{s}=2 s$ and $t_{e}=5 s$ which is sufficient to reach converged steady-state values), $N_{p}$ is the number of computational droplets injected into the domain (i.e., particle injection rate), $n_{i}$ is the number of real droplets represented by the single simulated droplet $i, w_{d, i}$ is the droplet velocity in the vertical direction and $V_{\text {sample }}$ is a sampling volume in which droplets are collected. The sampling volume is taken here as a sphere with a radius of $10 \mathrm{~mm}$. A similar approach is used in the experiments and applied to the number of detected droplets [11], except that the sampling volume is taken as $V_{\text {sample }}=A \times \operatorname{dof}$ where $A$ is the area of the field of the camera and $d o f$ is referred to as the depth of field, a quantity which depends on the droplet size.

The VMD is not post-processed directly in FDS. It is calculated here by: (1) obtaining a histogram of the number of particles per bin (i.e., a range of droplet diameters), (2) calculating the corresponding cumulative volume fraction (CVF) and then (3) determining the diameter for which CVF $=0.5$. The width of bin $i$ considered here is $\Delta D_{d, i}=D_{d, i}^{\max }-D_{d, i}^{\min }=1 \mu \mathrm{m}$. The CVF for a given bin $i$ is calculated as:

$$
C V F_{i}=\frac{\sum_{j=1}^{i} N_{i}\left(\pi \bar{D}_{d, i}^{3} / 6\right)}{\sum_{j=1}^{N_{b i n}} N_{i}\left(\pi \bar{D}_{d, i}^{3} / 6\right)}
$$


where $N_{b i n}$ is the number of bins, $N_{i}$ is the number of droplets collected in each bin $i$, and $\bar{D}_{d, i}$ is the average droplet diameter in a bin. The latter is calculated as $\bar{D}_{d, i}=\left(D_{d, i}^{\max }+D_{d, i}^{\min }\right) / 2$.

\section{RESULTS}

\section{Influence of particle injection rate}

A uniform volume flux angular distribution has been used for the simulations analyzed herein. The analysis is performed in terms of deviation between numerical and experimental data, which is defined as $\varepsilon=$ (numerical value - experimental value) / experimental value. Figure 5a shows, as suggested in [8], a linear increase in the deviation between the numerical simulations and the water volume flux measurement on the centreline as a function of $1 / \sqrt{N_{p}}$. This trend is observed for particle injection rates between $N_{p}=5 \times 10^{4} s^{-1}$ and $N_{p}=5 \times 10^{6} s^{-1}$. A constant value is observed for $N_{p}=5 \times 10^{3} s^{-1}$ and $N_{p}=1 \times 10^{4} s^{-1}$. The linear trend line in Fig.5a shows that an infinitely high number of injected Lagrangian particles per second (equivalent to $1 / \sqrt{N_{p}}=0$ ) would lead to a deviation of about $25.7 \%$. Such deviation could be interpreted as a systematic deviation that is not due to $N_{p}$-induced error but to other modelling aspects, such as for example gas phase turbulence modelling (and more specifically the turbulent viscosity model) or shortcomings in the inlet boundary condition for the spray, or other reasons. Therefore, the approach described herein can help the modeller distinguish the $N_{p}$-induced error from other sources of error.
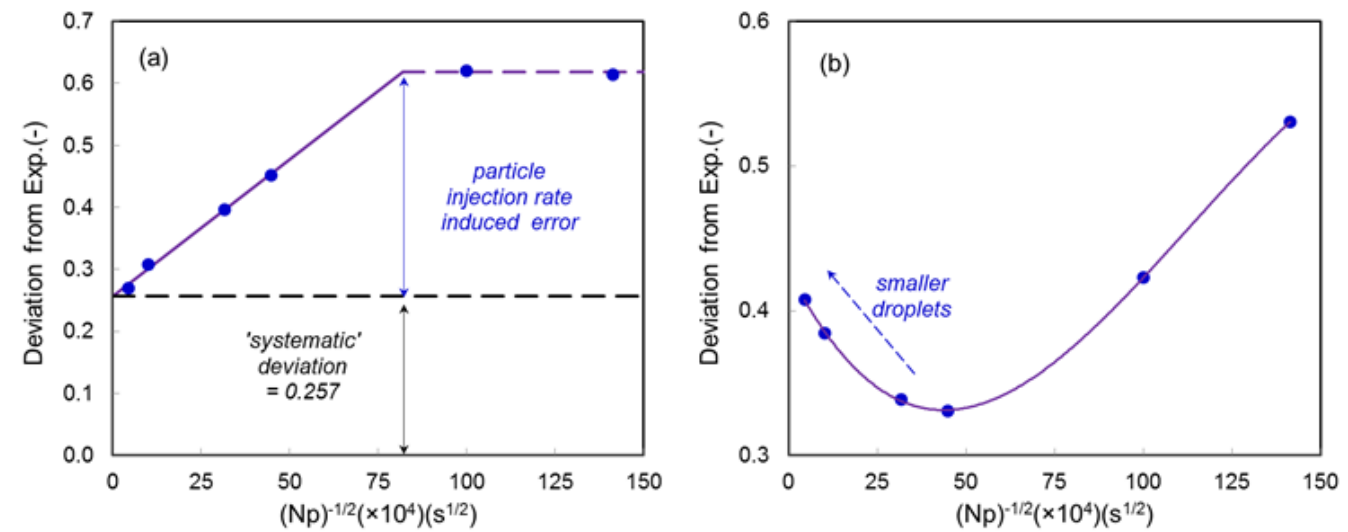

Fig. 5. Influence of the particle injection rate, $N_{p}$ on the deviation between experimental measurements and numerical predictions of the (a) water volume flux and (b) volume-median diameter on the centreline. The Rosin-Rammler distribution is used.

Figure $5 \mathrm{~b}$ shows, in a similar way to Fig $5 \mathrm{a}$, the obtained results for the volume-median diameter. However, the trends are different. More specifically, after reaching a minimum deviation of $33 \%$ from the experimental value at $N_{p}=5 \times 10^{4} s^{-1}$ (starting from lower $N_{p}$ values), the deviation starts to increase again for higher values of $N_{p}$ with a predicted VMD becoming smaller and smaller. As $N_{p}$ is increased, more small droplets are used to represent the volume flow rate delivered by the nozzle. This is believed to be a feature of the Rosin-Rammler distribution. In order to verify this assumption, the same simulations are re-run with a RosinRammler-lognormal distribution and analysed in the next section.

Figure 6 shows the influence of $N_{p}$ on the computational time (CPU). The latter parameter is made nondimensional using the minimum CPU, which has been obtained in this case for $N_{p}=1 \times 10^{5} \mathrm{~s}^{-1}$. Figure $6 \mathrm{a}$ cleraly shows, as expected, that the CPU generally increases as more Lagrangian particles are introduced in the computational domain. However, when we focus on the interval $5 \times 10^{3} \leq N_{p} \leq 1 \times 10^{5}$ (as displayed in Fig. 6b), we observe an opposite (counter-intuitive) trend where the CPU time increases as the particle injection rate gets lower. This can be explained by a longer time required to reach convergence in the numerical method when coupling the liquid phase and the gas phase. On Fig.6 the curve showing the deviation from the experimental measurement is also plotted. One can see (in Fig. 6a) that the slope of this curve, as opposed 

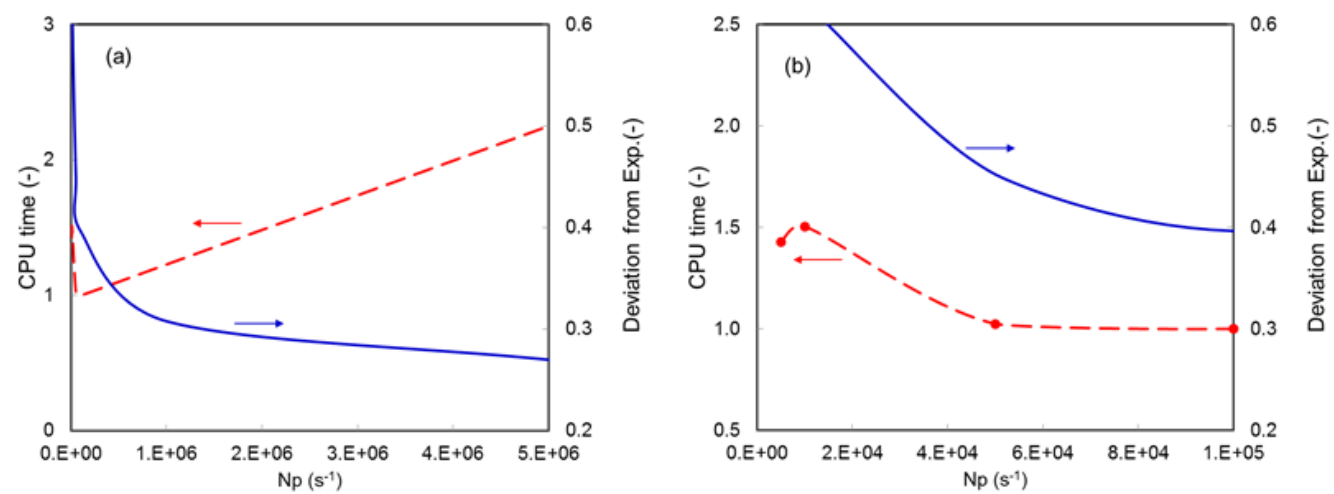

Fig. 6. Influence of the particle injection rate, $N_{p}$, on the computational time and the deviation between the experimental measurement and numerical predictions of the water volume flux on the centreline. The

Rosin-Rammler distribution is used.(a) Full range of $N_{p}$. (b) Focus on $5 \times 10^{3} \leq N_{p} \leq 1 \times 10^{5}$.

to the CPU curve, decreases with increasing $N_{p}$. This means that beyond a critical number $N_{p}$, an increased CPU time (associated to an increased $N_{p}$ ) does not warrant a significant improvement in the accuracy of the CFD simulation anymore, so the ratio CPU time/accuracy deteriorates.

\section{Influence of droplet size distribution}

The same sensitivity study performed above is undertaken here, except that the Rosin-Rammler-lognormal distribution is used instead of the Rosin-Rammler-distribution.
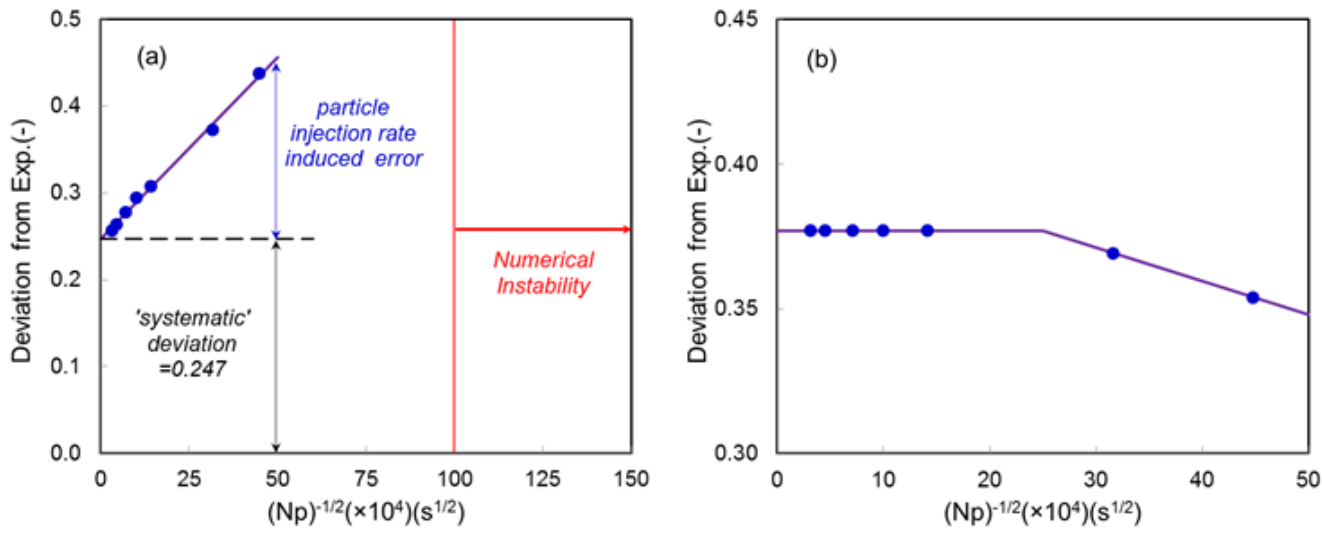

Fig. 7. Influence of the particle injection rate, $N_{p}$ on the deviation between experimental measurements and numerical predictions of the (a) water volume flux and (b) volume-median diameter on the centreline. The Rosin-Rammler-lognormal distribution is used.

The results displayed in Fig.7a are quite similar to the results displayed in Fig. 5a, although a numerical instability has been obtained when the particle injection rate is too low. The major difference lies in the results for the volume-median diameter. As formulated in the previous section, the numerical problem encountered when using the Rosin-Rammler distribution, and that prevented reaching convergence of the VMD in terms of $N_{p}$, is removed when the Rosin-Rammler-lognormal distribution is used. Figure $7 \mathrm{~b}$ shows indeed a very good convergence of the VMD, which remains constant above $N_{p}=5 \times 10^{5} s^{-1}$. The main trend for the CPU time displayed in Fig. 8a is similar to Fig. 6 for high $N_{p}$ values, but the peculiar behaviour observed for low values of $N_{p}$ is not encountered here. Based on the results obtained herein, we recommend using the RosinRammler-lognormal distribution. All results hereafter have been obtained with this distribution. Furthermore, a particle injection rate of $N_{p}=3 \times 10^{6} s^{-1}$ will be used, which, according to the results displayed in Fig. 8a 

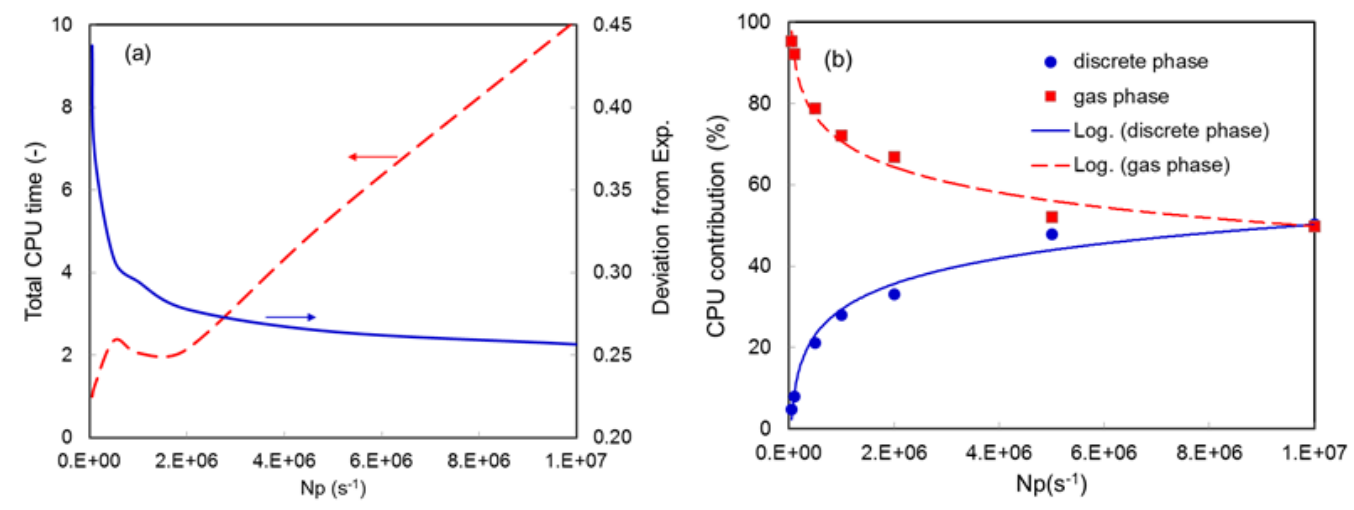

Fig. 8. Influence of the particle injection rate, $N_{p}$, on (a) the computational time and the deviation between the experimental measurement and numerical predictions of the water volume flux on the centreline and (b) the relative CPU contribution of both the discrete phase and the gas phase. The Rosin-Rammler-lognormal distribution is used.

provides a good compromise between accuracy and computational time. Figure $8 \mathrm{~b}$ shows the relative CPU contribution of the gas phase (resp. the discrete phase), which decrease (resp. increase) with increased values of $N_{p}$, following a logarithmic trend.

\section{Influence of the angular probability distribution}

To the best of our knowledge, the influence of the volume flow angular probability distribution on the spray pattern has not been thoroughly examined in the literature. In Fig. 9, the difference between a uniform distribution and a Gaussian distribution (with $\beta=1,5$ and 10) is visualized in terms of spray pattern shape and mean vertical gas velocity field. One can clearly see that the spray pattern shape induced by a Gaussian distribution could almost be assimilated to a reduction in the elevation angle, $\theta$. The higher $\beta$, the more reduction in angle is observed. Figure 10 shows that the deviation (in terms of centreline water volume flux) is below $10 \%$ when $\beta$ is between 1 and 2 . When $\beta$ is between 0 and 3, the deviation is kept below $30 \%$. As opposed to the work performed for the particle injection rate, $N_{p}$, which is a purely numerical parameter, it is difficult to find the best value for $\beta$ because the deviation from the experimental value could be induced by several other possible modelling parameters, including the cell size. This will be briefly addressed in the next section.

As mentioned in the introduction, it is generally assumed that more droplets are concentrated in the core region of the spray. This is done for example in FDS using the Gaussian function in Eq.(6) with $\beta=5$ as a default set-up [3,7]. In [3], the authors did not perform a sensitivity analysis on the angular probability distribution, but they did so for the elevation angle (called also in [3] the spray angle parameter). They found out in their study that narrowing the angle by 5 degrees increased the error by as much as $120 \%$, whereas increasing the angle by also 5 degrees resulted in significantly lower errors. This is expected given the Gaussian distribution that is used in [3]. The question that arises here is whether the angular probability distribution calibrated in [3] is suitable for the spray considered herein or not.

\section{Influence of the turbulent viscosity model and the cell size}

In Figs.5a and 7a a systematic deviation between experimental measurements and numerical predictions is estimated. As mentioned above, such deviation is not due to errors induced by the limited number of Lagrangian particles per second introduced into the computational domain (i.e., $N_{p}$ ). Rather, it is associated with uncertainties in other modelling aspects, such as the turbulent viscosity model and the cell size. 
(a) Uniform distribution
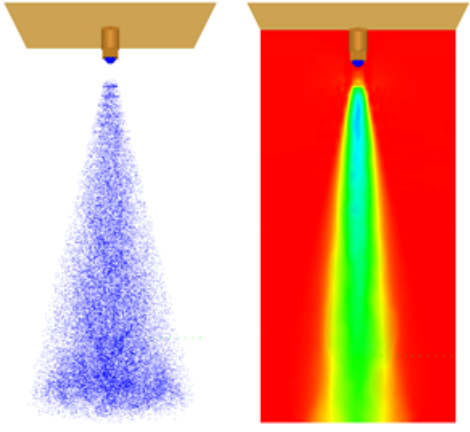

(b) Gaussian distribution $(\beta=5)$
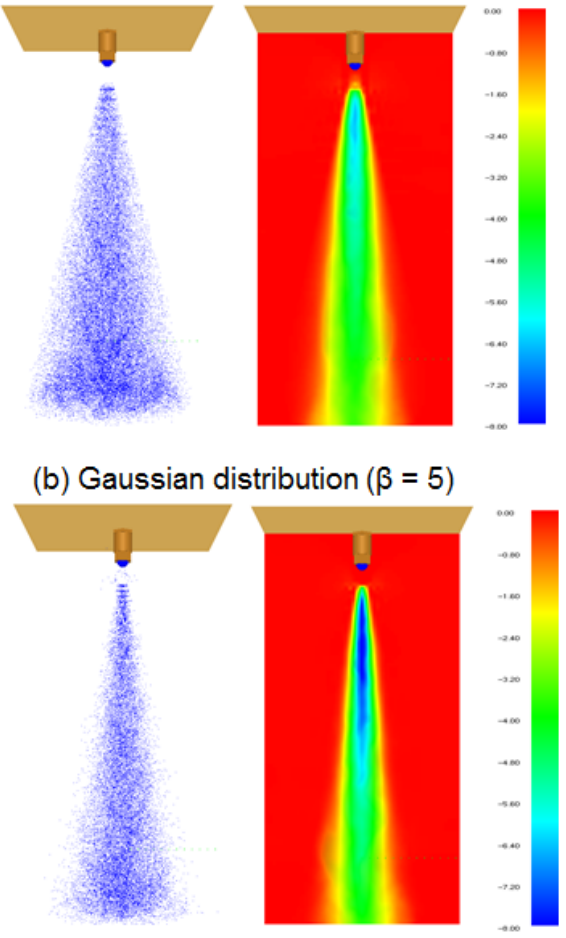

(b) Gaussian distribution $(\beta=1)$
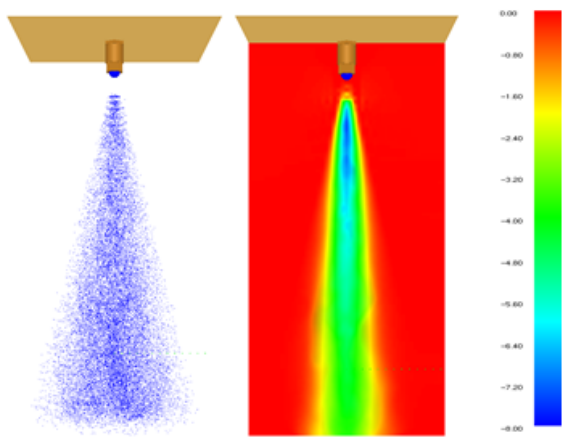

(b) Gaussian distribution $(\beta=10)$

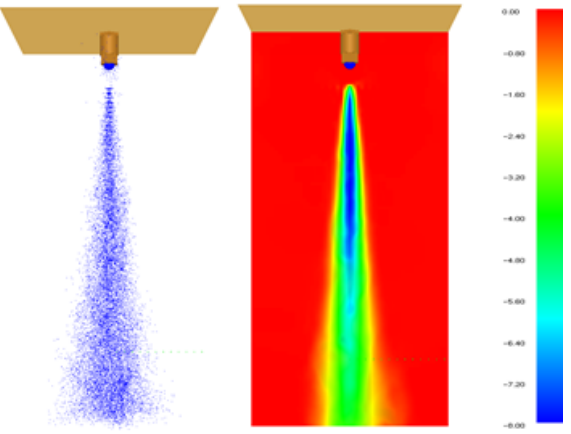

Fig. 9. Influence of the volume flux angular distribution on the spray pattern and the time-averaged gas velocity.

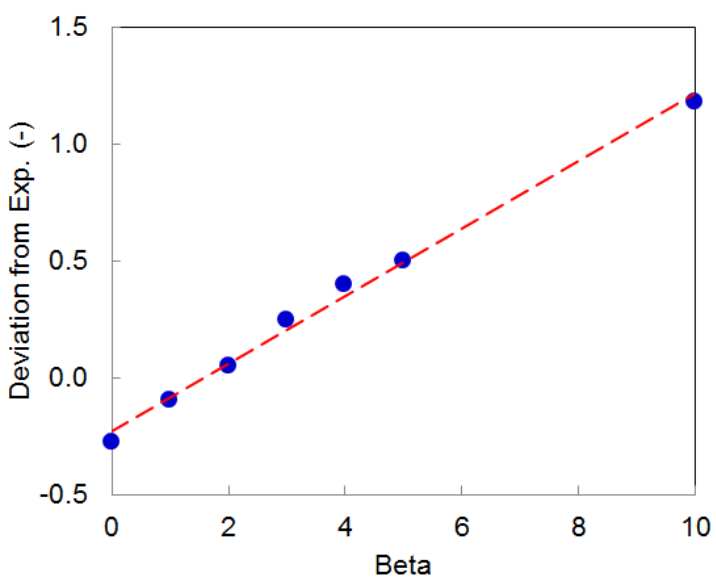

Fig. 10. Influence of the spray parameter $\beta$ (Eq.(6)) on the deviation between the experimental measurement and numerical predictions for the water volume flux on the centreline.

Figures 11 and 12 show the influence of the cell size on the radial profiles of the water volume flux and volume-median diameter using respectively the dynamic Smagorinsky model and the modified Deardorff model. Figures 11a and 12a show that, as noted in [3], both models converge to almost the same result using the finest grid. However, we further note here that the dynamic Smagorinsky model is more sensitive to the cell size than the modified Deardorff model. Using the former model led to a difference of about $100 \%$ for the volume flux on the centreline between the finest and the coarsest mesh. With the latter model this number is reduced to about $30 \%$. Examining the results displayed in Figs. 11b and 12b shows that the droplet size is less sensitive to the cell size, although a slight improvement is observed with the finest grid near the outer 

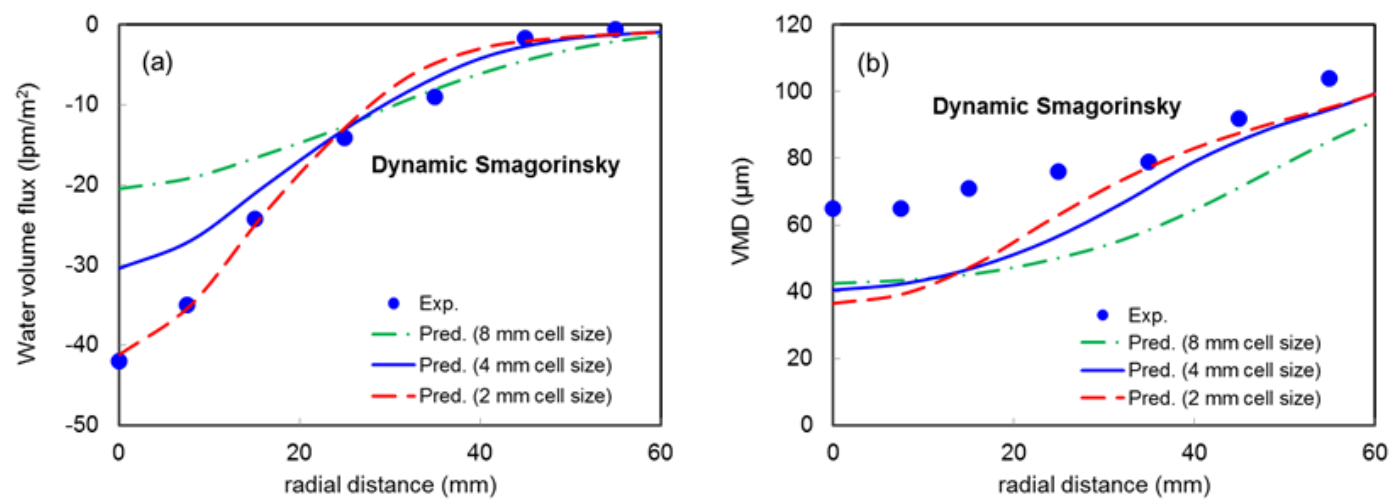

Fig. 11. Influence of the cell size on the radial profiles of (a) the water volume flux and (b) the volume-median diameter, using the Dynamic Smagorinsky model.
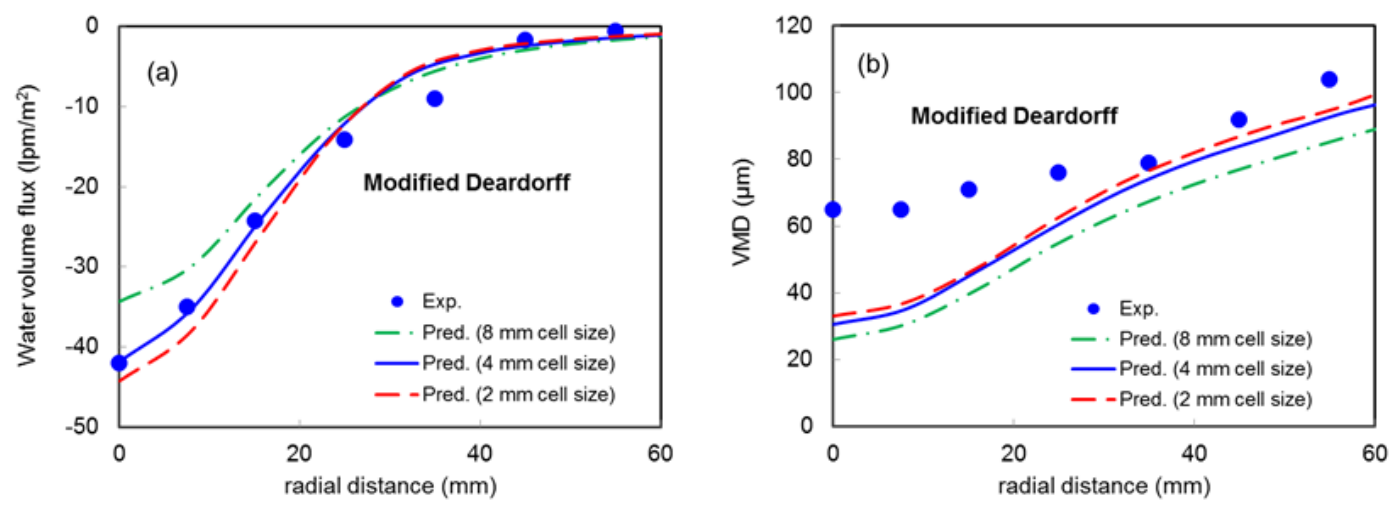

Fig. 12. Influence of the cell size on the radial profiles of (a) the water volume flux and (b) the volume-median diameter, using the Modified Deardorff model.

spray boundary. Furthermore, both the Smagorinsky and the modified Deardorff models result in a significant underprediction of the volume-median diameter in the core region of the spray. On the centreline the VMD predicted by the dynamic Smagorinsky (resp. the modified Deardorff) is about $40 \mu \mathrm{m}$ (resp. $30 \mu \mathrm{m}$ ), whereas the experimental value is $65 \mu \mathrm{m}$. In order to check if this underprediction could be attributed to a poor spray pattern resolution near the nozzle, we examined the water volume flux and VMD profiles not only in the far-field (at $300 \mathrm{~mm}$ from the nozzle) but also in the near field at $30 \mathrm{~mm}$ downstream the nozzle. The results displayed in Fig. 13 were obtained using the modified Deardorff model, a cell size of $4 \mathrm{~mm}$ and two values for the $\mathrm{C}$ factor, i.e., $\mathrm{C}=0.95$ and $\mathrm{C}=0.75$. These results do not show substantial differences between the two values of $\mathrm{C}$. However, the value of $\mathrm{C}=0.95$ provides a better agreement for the water volume flux profile in the far-field.

\section{CONCLUSION}

A detailed sensitivity analysis on the volume flux probability density function (PDF) used to represent and predict water spray patterns has been performed with the Fire Dynamics Simulator (FDS 6.1.2) for the water mist spray characterized in [1]. It is a $30^{\circ}$ full cone water mist spray emerging from a nozzle that operates at a pressure of $750 \mathrm{kPa}$ and delivers a water flow rate of $0.084 \mathrm{lpm}$.

The results show that, as noted in [8], the errors induced by the limited number of computational droplets are proportional to $1 / \sqrt{N_{p}}$ and could reach up to $35 \%$ (if $N_{p}$ is too low). However, the computational time (CPU) generally increases linearly with $N_{p}$. Nevertheless, if too low values of $N_{p}$ are employed in combination with the Rosin-Rammler distribution, an opposite trend (i.e., an increased CPU as a function of $N_{p}$ ) has been 

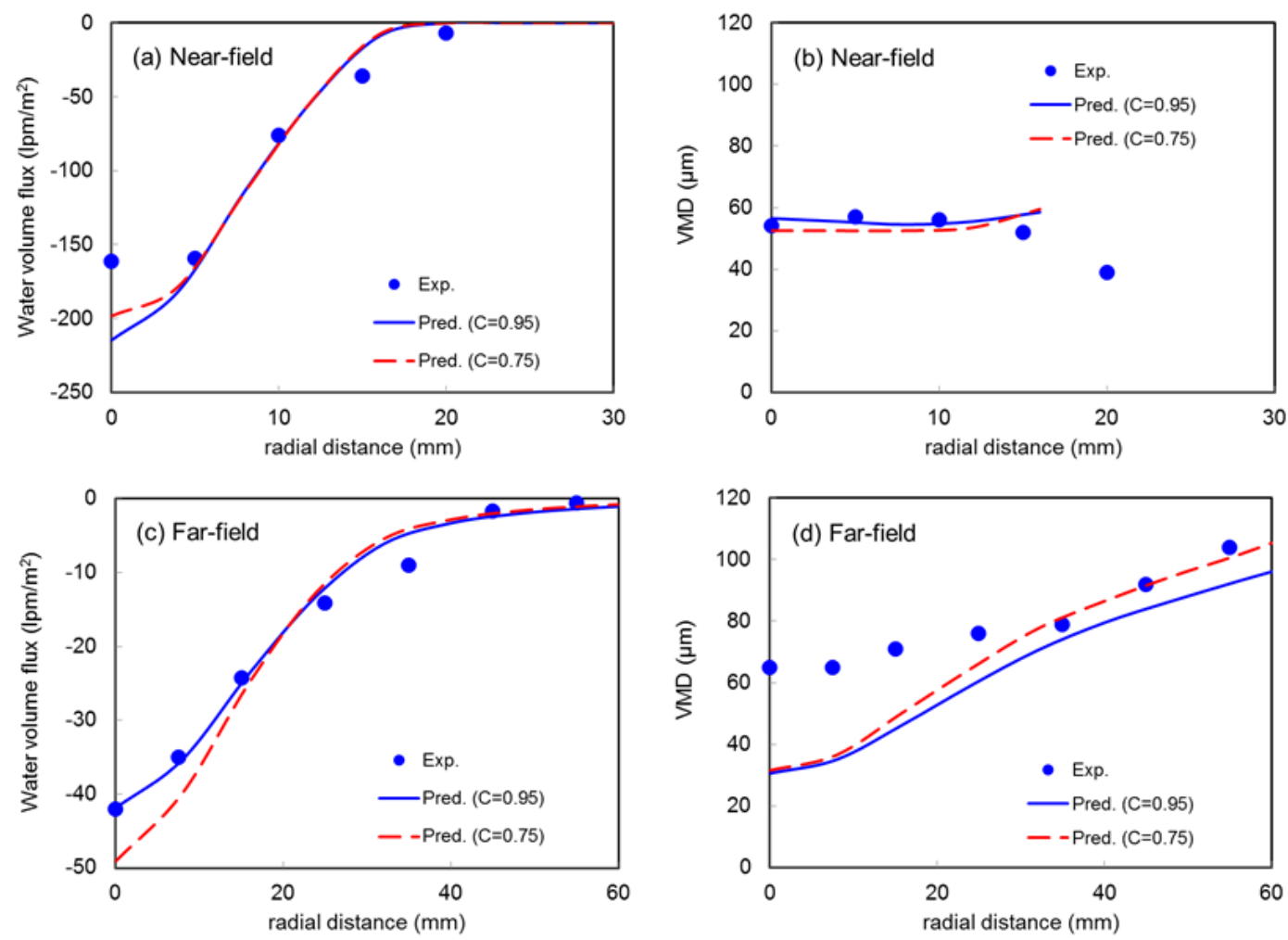

Fig. 13. Influence of the $\mathrm{C}$ factor on the near-field profiles (top figures) and far-field profiles (bottom figures) of water volume flux (left figures) and volume median-diameter (right figures).

illustrated and explained by a longer time required to reach convergence in the numerical method when coupling the liquid and the gas phase.

The theoretical background describing the Rosin-Rammler and the Rosin-Rammler-lognormal distributions for the droplet size demonstrates the limitations of the former in terms of connecting the droplet volume fraction to the droplet density function. This is illustrated by comparing the results in Figs. $5 \mathrm{~b}$ and $7 \mathrm{~b}$. As opposed to the Rosin-Rammler distribution, a converged volume-median diameter is obtained with the lognormal-Rosin-Rammler distribution when $N_{p}$ is increased.

A uniform angular distribution has been shown to provide a better agreement with the experiments than the Gaussian-type distribution (with more water concentrated in the core of the spray) prescribed in [3].

A sensitivity analysis on the cell size and the turbulent viscosity model shows that, as noted in [3], for a sufficiently fine grid (i.e., cell size of $2 \mathrm{~mm}$ ), the dynamic Smagorinsky and the modified Deardorff model converge to similar radial profiles of the water volume flux at $300 \mathrm{~mm}$ from the nozzle, with a deviation of less than $6 \%$ from the experiments. The dynamic Smagorinsky model was shown to be more sensitive to the cell size than the modified Deardorff model. Using the former model led to a difference of about $100 \%$ for the volume flux on the centreline between the finest and the coarsest mesh. With the latter model this number is reduced to about $30 \%$. The volume-median diameter was more difficult to predict than the water volume flux. Deviations with the experimental data reached up 50\% in the core region of the spray.

\section{ACKNOWLEDGMENTS}

Tarek Beji is a Postdoctoral Fellow of the Fund of Scientific Research-Flanders (Belgium) (FWO-Vlaanderen). 


\section{REFERENCES}

[1] Zhou, X. (2015) Characterization of interactions between hot air plumes and water sprays for sprinkler protection, Proceedings of the Combustion Institute, 35:2723-2729, http://dx.doi.org/10. 1016/j.proci.2014.05.078

[2] McGrattan, K., Hammins, A. and Stroup, D.(1998) "Sprinkler, Smoke and Heat Vent, Draft Curtain Interaction - Large Scale Experiments and Model Development," National Institute of Standards and Technology, NISTIR 6196-1, Gaithersburg, MD, 1998, 148 p.

[3] Sikanen, T., Vaari, J., Hostikka, S. and Paajanen, A.(2014) Modeling and Simulation of High Pressure Water Mist Systems, Fire Technology, 50:483-504, http://dx.doi.org/10.1007/ s10694-013-0335-8

[4] Marshall, A.W. and Di Marzo, M. (2004) Modelling Aspects of Sprinkler Spray Dynamics in Fires, Process Safety and Environmental Protection, 82:97-104, http://dx.doi.org/10.1205/ 095758204322972744

[5] Nam, S. (1996) Development of a computational model simulating the interaction between a fire plume and a sprinkler spray, Fire Safety Journal, 26:1-33, http://dx.doi.org/10.1016/0379-7112(96) 00015-x

[6] McGrattan, K., Hostikka, S., McDermott, R., Floyd, J., Weinschenk, C. and Overholt, K., "Fire Dynamics Simulator, User Guide," National Institute of Standards and Technology, NIST Special Publication 1019 6th Edition, Gaithersburg, MD, 2013, 262 p.

[7] McGrattan, K., Hostikka, S., McDermott, R., Floyd, J., Weinschenk, C. and Overholt, K., "Fire Dynamics Simulator, Technical Reference Guide, Volume 1: Mathematical Model," National Institute of Standards and Technology, NIST Special Publication 1018 6th Edition, Gaithersburg, MD, 2013, 149 p.

[8] Myers, T.M. and Marshall, A.W.(2016) A description of the initial fire sprinkler spray, Fire Safety Journal, 84:1-7, http://dx.doi.org/10.1016/j.firesaf.2016.05.004

[9] Bailey, A.G. (1983) The Rosin-Rammler size distribution for liquid droplet ensembles, Journal of Aerosol Science, 14:39-46, http://dx.doi.org/10.1016/0021-8502(83)90083-6

[10] Meredith, K.V., Zhou, X., Ebrahimzadeh, S. and Merci, B. (2015) Numerical Simulation of SprayPlume Interactions, 9th U.S. National Combustion Meeting, 2015.

[11] Zhou, X., DAniello, S.P. and You, H-Z.(2012)]Zhou:2012 Spray characterization measurements of a pendent fire sprinkler, Fire Safety Journal, 54:36-48, http://dx.doi.org/10.1016/j.firesaf.2012.07. 007 